\title{
Kernos
}

Revue internationale et pluridisciplinaire de religion grecque antique

3 | 1990

Varia

\section{La figure d'Apollon dans les principaux manuels de mythologie à la Renaissance}

\section{Monique Mund-Dopchie}

\section{OpenEdition}

\section{Journals}

Édition électronique

URL : http://journals.openedition.org/kernos/999

DOI : $10.4000 /$ kernos.999

ISSN : 2034-7871

Éditeur

Centre international d'étude de la religion grecque antique

Édition imprimée

Date de publication : 1 janvier 1990

ISSN : 0776-3824

Référence électronique

Monique Mund-Dopchie, « La figure d'Apollon dans les principaux manuels de mythologie à la

Renaissance », Kernos [En ligne], 3 | 1990, mis en ligne le 19 avril 2011, consulté le 03 mai 2019. URL http://journals.openedition.org/kernos/999; DOI : 10.4000/kernos.999 


\section{LA FIGURE D'APOLLON DANS LES PRINCIPAUX MANUELS DE MYTHOLOGIE À LA RENAISSANCE}

Bien que la culture antique des hommes du XVIe siècle ait été à la mesure de leur enthousiasme et de leur soif de connaissances, elle ne s'abreuvait pas nécessairement aux textes grecs et latins, sources principales de ce savoir. Dans le domaine de la mythologie notamment, les lettrés recouraient plus volontiers aux compilations érudites qui leur avaient été léguées par le Moyen Age ou procurées par de doctes contemporains. Car ils y trouvaient, déjà rassemblés et organisés, les multiples renseignements qu'il leur aurait fallu glaner, sinon, au cours de lectures interminables ${ }^{1}$. Ces traités contribuèrent ainsi, dans une mesure non négligeable, à former et à répandre dans le public une certaine image des dieux antiques. C'est pourquoi une étude consacrée à la figure d'Apollon dans la littérature et les arts de la Renaissance doit obligatoirement passer par une investigation du côté des mythographes.

Parmi les ouvrages les plus importants, on compte la Généalogie des Dieux de Boccace, composée vers le milieu du XIVe siècle ${ }^{2}$, dont le succès ne se dément pas aux siècles suivants, la Théologie mythologique de l'Allemand Georg Pictor, parue en 1532 et rééditée sous le titre Le magasin des Dieux en $1558^{3}$, l'Histoire des Dieux de Lilio Gregorio Gyraldi, publiée à Bâle en $1548^{4}$, la Mythologie de Natale Conti, publiée à Venise en $1551^{5}$, et les Images des Dieux de Vincenzo Cartari, publiées à

1 J. SEznec, La survivance des dieux antiques. Essai sur le rôle de la tradition mythologique dans l'humanisme et dans l'art de la Renaissance, Paris, 1980, p. 199 [1e éd., Londres, 1940].

2 G. BocCACE, Genealogie deorum gentilium libri, 1350-1360. Éd. V. Romano, 2 vol., Bari, 1951. J'ai utilisé un exemplaire de l'édition de Paris, 1511 (Louvain-laNeuve, CGD, Rés. 3B 2708).

3 G. PICtor, Theologia mythologica... in compendium congesta, Fribourg en B., 1532; Anvers, 1532 et Apotheseos tam exterarum gentium quam Romanorum deorum libri tres, Bâle, 1558. J'ai utilisé un exemplaire de la 1e édition (Bruxelles, BR, VII. 12.409 [a]).

4 L.G. GYRALDI, De deis gentium varia et multiplex historia, Bâle, 1548. Ce traité sera réédité dans des Opera omnia par Th. Guarinus à Bâle en 1580 (exemplaire consulté : Louvain-la-Neuve, CGD, Rés. BL 3485) et par J. Jensius à Leyde en 1696.

5 N. ConTI, Mythologiae sive explicationum fabularum libri decem, Venise, 1551. 
Venise en $1556^{6}$. Ces cinq traités furent très largement diffusés ${ }^{7}$; les deux derniers, particulièrement, connurent de nombreuses rééditions et traductions ${ }^{8}$. Pourtant leurs mérites n'étaient pas à la hauteur de leur réputation. Car ils ont révélé une mythologie boursouflée, livresque et barbare en accumulant les témoignages de toute provenance - fournis par les auteurs classiques, les écrivains de la basse Antiquité, les Pères de l'Église et les érudits médiévaux - et en faisant une large part aux divinités étrangères. Sans doute retenir toutes les sources accessibles était-il de bonne méthode; à condition toutefois de les aborder avec un sens aigu de la critique et de l'histoire. Hélas, nos mythographes en semblent totalement dépourvus ou, en tout cas, ne se sont guère préoccupés d'y recourir. Ils ont livré leur documentation en vrac, sans en apprécier le poids et sans l'organiser chronologiquement. Ils n'ont tenu compte ni des lieux ni des époques, ils ont mêlé les dieux d'où qu'ils viennent, les plus anciens et les plus récents, et ont privilégié ce qui leur paraissait bizarre, extraordinaire ${ }^{9}$. Aussi la représentation d'Apollon qui résulte de telles démarches n'a-t-elle plus grand chose à voir, comme nous l'observerons à l'instant, avec celle que nous offrent, par exemple, l'hymne homérique et la statuaire archaïque et classique.

On notera en premier lieu un manque d'intérêt assez flagrant pour la biographie d'Apollon. La plupart des mythographes se contentent, en

6 V. CARTARI, Le immagini colla sposizione degli Dei degli Antichi, Venise, 1556.

7 Sur l'influence exercée par ces manuels, voir notamment F.L. SCHOELL, Les mythologistes italiens de la Renaissance et la poésie élisabéthaine, in Études sur l'humanisme continental en Angleterre à la fin de la Renaissance, Paris, 1926, p. 21-42; DeWitt T. STARnes et E.W. TALBERT, Classical Myth and Legend in Renaissance Dictionaries. A Study of Renaissance Dictionaries in their Relation to the Classical Learning of Contemporary English Writers, Chapel Hill, 1955, passim; Mercure à la Renaissance, par Cl. Balavoine, W. Boerner, G. Demerson, Cl.-G. Dubois, G. Durand, M.-M. de La Garanderie, J.-F. Maillard, D. Menager, I. Pantin, L. Schrader, C. Vasoli, M. Vasselin, Paris, 1987, passim.

8 SEzNEc, p. 247, recense ainsi pour le livre de N. ConTi 16 rééditions et une traduction française de J. Montlyard, qui fut imprimée à 5 reprises. J'ai utilisé un exemplaire de l'édition latine de Francfort, 1596 (Louvain-la-Neuve, CGD, Rés. AL 17793) et un exemplaire de la traduction française de Lyon, 1607 (Louvain-la-Neuve, CGD, 3B 4856). L'ouvrage de CARTARI connut 12 éditions en italien, 5 éditions en latin, 5 éditions en français, 1 édition en anglais et 1 édition en allemand. J'ai utilisé un exemplaire de la traduction française, éd. de Lyon, 1581 (Bruxelles, BR, VB. 5963[B/1]). SEZNEC, op. cit., p. 199-228. 
effet, de maigres allusions à quelques épisodes de la vie du dieu, comme si la matière en était suffisamment connue et ne méritait pas de plus amples développements. Ils retiennent essentiellement, en raison de la haute teneur symbolique qu'ils leur attribuent, le meurtre du serpent Python et celui des Cyclopes, de même que le servage chez Admète auquel Apollon fut soumis ${ }^{10}$. Seul Natale Conti pousse l'enquête de façon plus systématique. Il évoque ainsi, outre les épisodes déjà mentionnés, la naissance merveilleuse à Délos, les nombreux fils du dieu, ses amours avec Hyacinthe et avec Daphné, sa participation, aux côtés de Laomédon, à la construction des murs de Troie, le concours de divination qui opposa Mopsos à Calchas et le meurtre du géant Tityos ${ }^{11}$. S'ils ne se soucient guère de raconter les aventures du dieu, les auteurs de manuels collectionnent, en revanche, avec un goût maniaque les détails érudits qui concernent la personne et la famille d'Apollon. Ils abordent tous sa généalogie et ses origines; mais, face aux versions discordantes qui en sont présentées, ils se contentent de distinguer, à la suite de Cicéron ${ }^{12}$, quatre Apollons. Les étymologies fantaisistes du nom remontent également aux sources anciennes, qu'il s'agisse de Macrobe, de Platon, des Stoïciens ou de Varron. Lilio Gyraldi, qui est le plus féru de ces matières, en dénombre au moins 7 , dont la plupart s'expliquent par la nature solaire du dieu. Plus sensible que les autres mythographes à l'onomastique, le même humaniste dresse en outre une liste de quelque 150 épithètes d'Apollon, dont il fournit plusieurs interprétations en recourant tantôt à l'étymologie, tantôt au culte, tantôt à la géographie, tantôt encore à l'apparence physique du dieu. Le relevé des animaux, des plantes et des objets associés au culte d'Apollon figure, lui aussi, à des degrés divers chez nos érudits. C'est Vincenzo Cartari qui explore le plus cette voie en rattachant au Seigneur de Delphes le loup, le corbeau, le cygne, l'épervier, le bœuf, le laurier, le trépied, pour ne citer que quelques exemples.

Enfin, les auteurs de manuels assimilent volontiers Apollon, conformément à la tradition syncrétiste si répandue dans l'Antiquité, à des divinités orientales telles que Horus, Osiris et Mithra. Ces amalgames témoignent d'un goût très prononcé pour l'exotisme et marqueront l'iconographie de la Renaissance ${ }^{13}$. Ainsi l'Apollon

10 BocCACE, ff. XLv-XLIr; PICTOR, ff. 12r-16r; GyRALdi, Syntagma VII; CARTARI, p. 54-104.

11 ConTI, Mythologiae, p. 344-370; Mythologie, p. 314-338.

12 Cicéron, De nat. deorum, ПII, 57.

13 SEZNEC, op. cit., p. 211-214. 
assyrien de Macrobe ${ }^{14}$ - et celui-ci n'est qu'un cas parmi d'autres - est décrit longuement par Georg Pictor, puis par Cartari, lequel en fait graver une illustration, paraphrasée de la sorte :

Les Assyriens monstrent la puissance que le Soleil a en ce monde, et les effects qu'il y fait, par une image d'Apollon, qui avoit la barbe longue et pointue, portant certain engin sur la teste semblable à un panier... Il estoit en apres vestu d'un corselet : et tenoit de la main droite une pique qui avoit à la cime une petite figure de la Victoire, et de la main gauche presentoit un bouquet de fleurs. Il avoit sur les espaules un drap en forme de manteau, ouvert devant, avec la teste de Meduse, environnée de serpens : à son costé estoient aucunes aygles, qui sembloient voler, et au devant ses pieds, une image de femme, laquelle de l'un et de l'autre costé avoit deux autres images pareillement de femme, qu'un grand serpent à plusieurs tours tortus enlaçoit. Ainsi descrit Macrobe ce simulacre15.

Cette gravure et cette description de Cartari ont influencé les artistes de l'époque, comme en témoigne un dessin exécuté pour la Mascarade de la Généalogie des Dieux, conservé au Musée des Offices à Florence. La primauté des reconstitutions d'après des renseignements écrits, face aux monuments figurés accessibles à la Renaissance, se trouve ainsi confirmée ${ }^{16}$.

Cette curiosité pour les images d'Apollon n'est pas gratuite. Elle rejoint en réalité une préoccupation essentielle de nos auteurs, à savoir les effets de sens, le message caché du mythe. Car, à l'instar des penseurs médiévaux, dont ils se révèlent ainsi les héritiers, ils préfèrent "saisir les phénomènes sous une forme complètement dématérialisée, comme des allégories, et les interpréter sur un plan transcendantal après les avoir rattachés au savoir spéculatif» ${ }^{17}$. Ce que le dieu Apollon signifie au point de vue moral, astral ou cosmique intéresse donc infiniment plus que le dieu Apollon lui-même.

Boccace - mais il sera pratiquement le seul - se présente à ce propos comme un disciple d'Évhémère, pour qui «les dieux traditionnels étaient simplement des souverains que la gratitude ou l'adulation de leurs sujets avaient élevés jusqu'aux cieux» ${ }^{18}$. Avec le compilateur

14 MACroBe, Saturnalia, I, 17, 66-67.

15 CARTARI, op. cit., p. 79-80.

16 SEZNEC, op. cit., p. 226.

17 E. KANTOROwICZ, L'Empereur Frédéric II, traduit de l'allemand par A. KoHN, Paris, 1987, p. 310-311.

18 SEZNEC, op. cit., p. 17. 
médiéval Théodontius, il voit en Apollon tantôt un roi-législateur (nomios), qui provoqua par sa sévérité la révolte de ses sujets et fut contraint de se réfugier auprès du roi Admète ${ }^{19}$, tantôt, et de préférence, un primus inventor, le premier médecin en l'occurrence, dont l'art englobe tous les talents régulièrement attribués au dieu. C'est parce qu'il connaît l'harmonie des mouvements humains qu'Apollon découvre l'harmonie du jeu de la cithare et la musique; c'est parce qu'il prédit la mort ou le salut à ses malades qu'il est doté de divination; c'est enfin parce qu'il donne des bons conseils à ses patients qu'il incarne splendidement la sagesse.

La tradition historique dans laquelle s'inscrit Boccace après les Pères de l'Église et plusieurs compilateurs médiévaux ne jouit cependant pas de la faveur des quatre autres mythographes. Elle est largement supplantée chez eux par la tradition physique, elle aussi attestée à travers l'Antiquité et le Moyen Age, qui consiste à diviniser les planètes et les astres et, dans notre cas, à assimiler Apollon au Soleil. Même Boccace s'en fait l'écho et l'interprétation que Cartari nous offre de l'Apollon assyrien, à la suite de Macrobe ${ }^{20}$, est significative à cet égard :

La barbe, qui pend en bas sur la poitrine, signifie, que le Soleil espand du ciel ses rayons en la terre. Le panier doré qui monte en haut, monstre le celeste feu, de quoy lon croit que le Soleil soit fait. La pique et la cuyrasse signifient Mars, car on dit que par luy est demonstrée la vehemente ardeur du Soleil. La Victoire veut à dire que tout est subject à la vertu du Soleil. La fleur denote la beauté des choses, lesquelles la secrete vertu du Soleil ensemence, comme de sa temperée chaleur, fait naistre, nourrit et conserve. La femme qui se tient devant ses pieds, est la terre, laquelle est decorée par les rayons que du ciel le Soleil espand dessus elle... Les autres deux femmes qui sont aux costez de celle là du milieu, signifient la matiere, dont sont faites les choses, et la nature, qui les fait : lesquelles paroist, que servent ensemble à la terre, faisans tant pour son embellissement. Le serpent qui les enlace, nous donne à entendre le chemin tortu que fait le Soleil. Les Aygles, parce qu'elles volent treslegerement, et en haut, signifient la hauteur, et la vistesse du Soleil. Fut apres adjousté le manteau sur les espaules et teste de Meduse, qui est l'escusson et marque de Minerve, pourautant que, comme dit Porphyre, Minerve

19 Ce type d'interpretation figure aussi chez ConTI, Mythologiae, p. 350; Mythologie, p. 320.

20 MacroBe, Satumalia, I, 17, 68-70. 
n'est autre, que ceste vertu du Soleil, laquelle esclaire les entendemens humains, et envoie la prudence dans les ames des mortels 21 .

Mieux que sa qualité de primus inventor, le symbolisme solaire d'Apollon permet, en effet, aux auteurs des traités mythologiques de structurer, dans une certaine mesure, des informations disparates. Il explique d'abord différents rôles conférés au dieu. Celui-ci révèle la vérité parce qu'il est la lumière qui succède à la nuit ou, si on préfêre les termes utilisés par Natale Conti ${ }^{22}$, parce qu'il chasse toutes les ténèbres et obscuritez de l'esprit de l'homme. Il fonde la médecine grâce à la chaleur qu'il dispense aux plantes; or les herbes qui croissent à l'abri sont beaucoup plus nuisibles que celles qui viennent à l'ombre, ou qui sont nourries en lieux eveux et humides. De même, on lui attribue l'invention de la musique de par sa situation au beau milieu des autres planetes, comme leur seigneur et prince, desquels les Pythagoriens ont creu que les mouvemens rendoient un concert et harmonie merveilleusement douce et agreable.

Certains épisodes de la vie d'Apollon revêtent également une signification précise dans cette perspective solaire. La victoire sur le serpent Python devient celle de la lumière sur le manque de foi, d'après Boccace, qui mêle de la sorte astronomie et morale. Pour Conti et Cartari, elle représenterait plutôt, comme le meurtre des Cyclopes d'ailleurs, la toute-puissance du soleil, éliminant les vapeurs de la terre et purgeant celle-ci de ses humidités malsaines. Quant aux animaux associés au culte d'Apollon, ils participent, bien entendu, à la nature solaire du dieu, ainsi que Cartari entreprend de nous le démontrer. Le loup d'Apollon-Lycée, qui voit dans la nuit, triomphe des ténèbres, à l'instar de son maître; le cygne lui est étroitement apparenté par sa blancheur lumineuse, le coq, par le chant matinal qui salue le lever du jour; l'épervier, lui, symbolise Apollon, parce que sous d'autres cieux, il évoque Osiris, le dieu-soleil des Égyptiens ${ }^{23}$. La plupart de nos mythographes pratiquent d'ailleurs volontiers le syncrétisme entre le dieu de Delphes, Horus ou Mithra, car tous trois nous renvoient au Soleil.

Enfin, à l'instar de l'Apollon assyrien, les différentes «images» véhiculées par les cinq manuels sont interprétées en fonction des propriétés du soleil. Le portrait dressé par Albricus, l'auteur du traité médiéval Liber Ymaginum Deorum, et repris par Boccace, Pictor,

21 CARTARI, op. cit., p. 80-82.

22 Cont, Mythologie, p. 335.

23 CARTARI, op. cit., p. 65. 
Gyraldi et Conti est exemplaire à cet égard. L'éphèbe élégant qui nous est dépeint évoque, on s'en serait douté, l'éternelle jeunesse du soleil, tandis que la couronne de 12 gemmes qui ceint son front rappelle les 12 signes du zodiaque. Quant aux flèches et aux Grâces qu'il tient dans les mains, elles symbolisent, les unes, la peste, les autres, le salut et représentent le pouvoir des rayons du soleil, dont la chaleur tantôt répand les virus des épidémies, tantôt permet de surmonter les maladies et met en fuite l'insalubrité2 24 .

Le symbolisme solaire d'Apollon se trouve donc accommodé à toutes les sauces et il envahira, de longues années encore, le domaine de l'art et celui de la littérature. On songe entre autres aux nombreuses représentations de Louis XIV, le roi-Soleil, sous les traits d'Apollon dans toute sa gloire lumineuse 25 . La vigueur de cette tradition n'implique pas pour autant une véritable adhésion de nos humanistes à l'astrologie et à ses implications. Au contraire, ceux-ci affichent l'orthodoxie de leur pensée en dénigrant de quelques phrases dédaigneuses des superstitions qu'ils se plaisent à raconter ou en proclamant leur intention de les interpréter comme des allégories morales. Nous n'en voulons pour preuve que ces avertissements réitérés de Natale Conti et de Vincenzo Cartari :

Nous avons souvent dict que les anciens ont donné les noms de divers Dieux aux forces et vertus de nature ou des astres, ou mesme aux actions par lesquelles Dieu besongne és affaires de ce monde... Et ne pense point que les anciens forgeans telles Fables aient eu autre intention ou sujet, sinon qu'adorans tantost les proprietez et vertus des elemens, tantost les estoilles en guise de Dieux, ils ont voulu par tels contes exalter la puissance de leurs Dieux. Car les Fables qui sont faites touchant les Dieux des Paiens, concernent la consideration des choses naturelles ou astronomiques : et celles qui sont faites touchant les hommes, servent pour dresser la vie humaine, et l'amender de mieux en mieux 26 .

Les opinions ayans esté diverses envers les anciens du commencement des choses, tant de la matiere que de l'auteur et createur d'icelles : les Poetes, lesquels furent les premiers, comme dit Aristote, qui escripvirent des Dieux, faignirent diverses fables d'iceux, faisans croire à l'abusée et sotte gent, que plusieurs furent

24 BoCCACE, f. XLIr; PICTOR, f. 12v; GYRALDI, p. 212; CONTI, Mythologiae, p. 368 et Mythologie, p. 337.

25 Cf. J.-P. NéraUdau, L'Olympe du Roi-Soleil, Paris, 1986, p. 51-52, 59, 68 et passim.

26 ConTI, Mythologie, p. 334 et 338. 
lesquels appellans Dieux les premiers inventeurs des choses, et des principales matieres d'icelles, exprimerent les divers advis et opinions des diverses sectes. Et contant des fables en ceste sorte, feirent Dieux les Elemens, les Estoilles, le Soleil et la Lune...27.

Ils affichent donc un scepticisme de bon aloi, scepticisme qu'ils attribuent aux Anciens eux-mêmes et qui les dédouane de répandre des savoirs suspects, voire de les étaler avec complaisance. Ils appartiennent donc pleinement au siècle d'un Aeneas Sylvius Piccolomini qui, en tant que pape, refusait de "paganiser" en littérature, mais qui se laissait entraîner par sa formation d'humaniste à encombrer ses propres écrits de réminiscences mythologiques ${ }^{28}$.

Dans une telle optique, on ne peut guère espérer que le domaine de la mantique excite la curiosité des mythographes et leur découvre des pistes à explorer. Ici encore on ne trouve que remarques faites au hasard, idées lancées à l'emporte-pièce ou digressions érudites. Nous pouvons regrouper les unes et les autres autour de trois axes principaux ${ }^{29}$. En premier lieu, il arrive à certains de nos humanistes d'intégrer la mantique dans l'interprétation globale qu'ils donnent d'Apollon. Ainsi, pour Boccace, la divination, nous l'avons vu, relève de la médecine, Apollon, premier médecin, prédisant à ses malades une issue fatale ou la guérison ${ }^{30}$. Mais le même Boccace explique également par la nature solaire du dieu sa capacité à prophétiser les maux qui attendent les hommes et il sera suivi dans cette voie par Natale Conti ${ }^{31}$.

En second lieu, avec une remarquable unanimité, les auteurs des manuels rattachent à la vocation prophétique et aux oracles d'Apollon la présence de certains animaux et végétaux qui lui sont régulièrement associés. Le laurier est mentionné à cinq reprises parce qu'il permet de connaître l'avenir, soit en provoquant des songes, lorsqu'on le place sous son oreiller, soit en crépitant de façon peu banale lorsqu'on le

27 CARTARI, op. cit., p. 54.

28 SEZNEC, op. cit., p. 233-242, et notamment p. 235.

29 À cela s'ajoutent, chez GYRALDI, quelques allusions aux oracles, que l'on trouve dans ses interprétations de certaines épithètes d'Apollon, Clarius, Grynaeus, etc.

30 BOCCACE, f. XLv.

31 BoCCACE, f. XLIr; CoNTr, Mythologiae, p. 368; Mythologie, p. 337; vague allusion chez CARTARI, op. cit., p. 70. 
brûle ${ }^{32}$. Le corbeau est régulièrement décrit comme un oiseau prophétique, car les variations dans ses croassements annoncent le soleil ou la pluie ${ }^{33}$. Le cygne, évoqué par Conti et par Cartari, est proche d'Apollon par sa blancheur, certes, mais aussi parce que, sentant sa mort venir, il entonne son plus beau chant ${ }^{34}$. Et Cartari de lui adjoindre le coq, animal solaire et prophétique tout à la fois, puisque son cocorico précède le lever de l'astre ${ }^{35}$.

Enfin, les techniques divinatoires font l'objet de longues digressions chez Gyraldi comme chez Conti. Le premier commence par rapporter quelques anecdotes piquantes, empruntées à Jamblique, Origène, Jean Chrysostome et d'autres ${ }^{36}$, sur la façon dont la Pythie vaticinait, avant de nous fournir - sans commentaire - une série impressionnante de techniques et de nous prévenir qu'il s'agit là uniquement de superstitions : superstitiosis vaticinantium generibus ${ }^{37}$. Le second nous livre une liste de procédés divinatoires, qu'il sait incomplète, mais ne manifeste pas le même dédain pour ceux-ci. Il relève leur exactitude, du moins lorsqu'ils ont trait au Christ. Pour Conti, en effet,

il ne faut point faire d'estat de ces devinemens, non plus que de chose de néant et pleine de vanité, et de tromperie, sur tout de ceux qui se font par physionomie, osselets, cercles, terre, crible, feu, bassin, eau, main, fromage, et rappel des morts. Néantmoins les Oracles n'ont pas laissé de predire et d'exprimer quelquefois la verité, comme ne pouvans pas tousiours tromper les hommes : comme aussi les Sibylles ont esté trouvées veritables en beaucoup de choses : et mesme Apollon a fort bien annoncé la mort de nostre Seigneur Jesus Christ, et la Sibylle plusieurs poincts concernans sa venue, nativité et miracles qu'il devoit faire devant sa mort et passion 38 .

32 BocCaCe, f. XLv; PiCToR, ff. 14v-15r; ConTi, Mythologiae, p. 355 et 368; Mythologie, p. 324-325 et 337; CARTARI, op. cit., p. 69-70; vague allusion chez GYRALDr, op. cit., p. 207 [=211].

33 BOCCACE, ff. XLv-XLIr; PICTOR, f. 15v; CARTARI, op. cit., p. 64; vague allusion chez GYRALDI, $i b$.

34 ConTI, Mythologiae, p. 359; Mythologie, p. 328; CaRTARI, op. cit., p. 64.

35 CARTARI, op. cit., p. 65.

36 Jamblique, De mysteriis, III, 11 (126); ORIGÈne, Contra Celsum, VII, 3; JEAN ChrYsostome, In Epist. I ad Cor. Hom. XXIX, 1; cf. aussi PluT., De defectu oraculorum, $414 \mathrm{~d}-\mathrm{e}$.

37 GYRALDI, op. cit., p. 220.

38 ConTI, Mythologie, p. 327; cf. aussi Mythologiae, p. 358. 
On vérifie, une fois de plus, la crédulité des mythographes. Comme bon nombre de contemporains et d'illustres prédécesseurs - Saint Augustin et Lactance - Conti croit à l'authenticité du Corpus Hermeticum, des Hieroglyphica d'Horus Apollo, des Oracles Sibyllins, et il admire en toute logique les convergences entre les traditions païenne et chrétienne ${ }^{39}$.

Les informations véhiculées par nos cinq manuels sur le dieu Apollon et sur ses oracles ne constituent donc en aucun cas une nouveauté. Elles s'inscrivent en droite ligne dans le prolongement du Moyen Age et de la basse Antiquité avec leurs amalgames douteux, leur érudition désordonnée, leur absence de perspective chronologique, leur goût prononcé pour l'allégorie et le symbole. Mais on ne peut en tenir rigueur à leurs auteurs. Car ils viennent trop tôt pour exploiter valablement l'apport original de la Renaissance. Ce n'est que dans la seconde moitié du XVIe siècle, en effet, que les humanistes disposent, grâce à l'imprimerie, de la quasi-totalité des textes grecs et latins hormis ceux qui seront fournis plus tard par les papyri- et des merveilleux outils que constituent le Thesaurus d'Henri Estienne et de solides manuels en différents domaines. À ce moment-là, mais à ce moment-là seulement, les sources du savoir seront confrontées et s'éclaireront mutuellement. Ces nouvelles données n'engendreront pas nécessairement de nouvelles questions, mais elles transformeront des vieilles réponses ${ }^{40}$. La science mythologique, comme les autres disciplines, bénéficiera à terme de cette évolution.

Collège Érasme

Monique MUND-DOPCHIE

Place Blaise Pascal, 1

B - 1348 LOUVAIN-LA-NEUVE

39 Voir à ce sujet A. GRAfTon, Higher Criticism Ancient and Modern : The Lamentable Deaths of Hermes and the Sibyls, in The Uses of Greek and Latin. Historical Essays, éd. A.C. DionisotTi, A. GraFTon et J. KraYe, Londres, 1988 (Warburg Institute Surveys and Texts, 16), p. 155-170.

40 GRAFTON, art. cit., p. 159-160 et 169-170. 


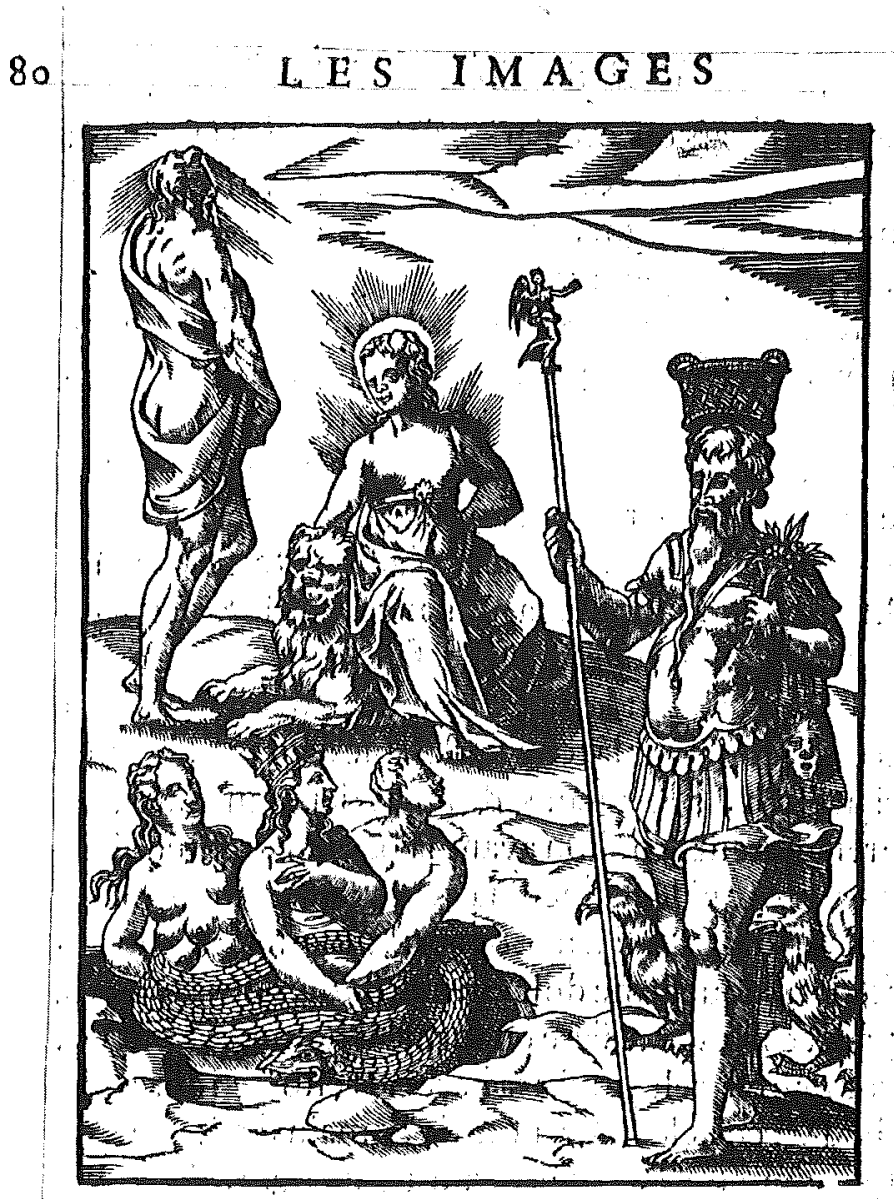

me qu'vin grand ferpent à plufieurs tours tortus enlaçoit. Ainfi defcrit Macrobe ce fimulacre, \& linterExpofition prete aufsicomme s'enfuit. Labarbe, qui pend en bas fur la poitrine, lignifie, que le Soleil efpand du ciel fes rayons en la terre. Le panier doré qui monte en haut, monftre le celefte feu, dequoy lon croit que le Soleil foit fait. La pique \& la cuyraffe fignifiend Mars.

Vincenzo CARTARI, Images, Lyon, 1581, p. 80.

Copyright Bibliothèque Royale, Bruxelles. Réserve précieuse : VB.5963 (B/1). 